\title{
Esclerosis múltiple recurrente remitente en el sector público de salud de Chile. Descripción clínica de 314 pacientes
}

\author{
JORGE NOGALES-GAETE ${ }^{1,2}$, RODRIGO ARACENA ${ }^{1,2}$, \\ SERGIO CEPEDA-ZUMAETA ${ }^{1,2}$, CLAUDIO ELOIZA ${ }^{1,2}$, \\ PAULA AGURTO ${ }^{1,2}$, VANNIA DÍAZ ${ }^{1, \mathrm{a}}$, SILVIA LABBÉ ${ }^{1, \mathrm{~b}}$, \\ SÉFORA MARTÍNEZ ${ }^{1, \mathrm{c}}$, JAZMÍN FLORES $^{1,4, \mathrm{~d}}$, CASANDRA ARAYA $^{3, \mathrm{~d}}$
}

\section{Clinical features of 314 patients with relapsing-remitting multiple sclerosis}

Background: Immunomodulatory drugs (IMD), Interferon $\beta 1 a, \beta 1 b$ and glatiramer acetate are available in the Chilean public health system since June 2008 for patients with relapsing-remitting multiple sclerosis (RR-MS). Diagnostic confirmation and programmed follow up of these patients is carried out at a public national reference center. Aim: To describe the epidemiological and clinical features of 314 patients evaluated in this center between 2008 and 2012. Patients and Methods: Review of clinical records, to obtain information about demographic background, medical history, expanded disability status scale of Kurtzke (EDSS), multiple sclerosis functional composite (MSFC), intensity fatigue scale of Krupp, Rao's Brief Repeatable Battery of Neuropsychological Tests (BNR-R) and anxious-depressive manifestations using Hamilton and Beck questionnaires. Results: The ages of patients ranged from 12 to 63 years and $67 \%$ were women. The initial symptoms were sensory disturbances in $20 \%$, motor alterations in $18 \%$ and optical neuritis in $16 \%$. In 9\% of patients, the disease began with several manifestations. The EDSS was 4 or less in $73 \%$ of patients and cognitive impairment was observed in $34 \%$. Treatment failure during the first and second years, occurred in 23 and 26\% of patients, respectively. Male gender, age under 40 and brainstem malfunction at the onset of disease, were predictive of treatment failure during the second year. Conclusions: The features of these patients are very similar to those reported abroad.

(Rev Med Chile 2014; 142: 559-566)

Key words: Disease progression; Immunomodulation; Multiple Sclerosisi, Relapsin-Remitting.
'Servicio de Neurología, Complejo Asistencial Barros Luco Trudeau. 2Departamento de Neurología Facultad de Medicina Universidad de Chile.

${ }^{3}$ Escuela de Fonoaudiología, Universidad Autónoma de Chile. ${ }^{4}$ Escuela de Fonoaudiología, Universidad Andrés Bello.

Santiago de Chile.

apsicóloga.

bEnfermera Universitaria.

'Kinesióloga.

dFonoaudióloga.

Recibido el 15 de diciembre de 2013, aceptado el 8 de mayo de 2014.

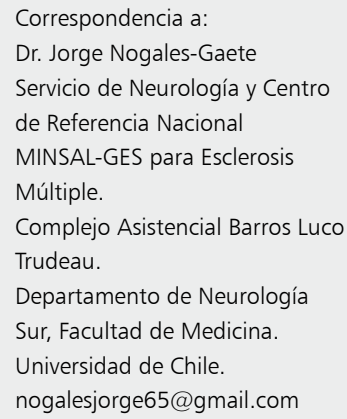

4 sclerosis múltiple (EM) es una enfermedad desmielinizante-degenerativa del sistema nervioso central (SNC). Es multifactorial, afecta individuos en etapas productivas y mayormente mujeres. Su prevalencia fue descrita por Davenport y Kurtzke, postulando en el hemisferio norte una distribución casuística latitudinal con distancia correlativa al Ecuador ${ }^{1-3}$. Su diagnóstico se basa en demostración clínica o para-clínica de diseminación témporo-espacial de lesiones desmielinizantes del SNC ${ }^{4}$. La evolución más frecuente es recurrente-remitente (EM-RR) caracterizada por episodios de disfunción neurológica mayores a 24 h de duración, denominados brotes 5 .

Las terapias se categorizan como dirigidas al manejo del brote, alivio sintomático y modificadoras de la evolución (FIM) ${ }^{6}$. En estas, en las denominadas terapias de primera línea, se ubi- 
can interferón-beta y acetato de glatiramer, (no accesibles en sistema público de salud chileno hasta 2008), y las de segunda línea, natalizumab y fingolimod, aún no disponibles.

Desde marzo de 2008, la calificación inicial y control programado de pacientes con EM-RR beneficiarios del sistema público chileno se realiza por nuestro equipo como referentes nacionales, primero a cargo de un programa piloto de FIM y posteriormente en el marco del sistema de garantías explicitas de salud (GES). Esto nos ha permitido evaluar una casuística de la que comunicamos aspectos clínico-epidemiológicos, basales y evolutivos. En este contexto, compartimos la opinión de Vizcarra respecto a que la divulgación de descripciones como esta sirve para el conocimiento local de la enfermedad ${ }^{7}$.

\section{Pacientes y Método}

Los pacientes fueron evaluados en el complejo asistencial Barros Luco (CABL) entre marzo de 2008 y julio de 2012. Evaluamos 407 pacientes, 314 de los cuales calificaron para tratamiento inmunomodulador. Realizamos: revisión de antecedentes demográficos, evaluación clínica neurológica, aplicación escala ampliada de discapacidad de Kurzke (EDSS), escala funcional compuesta, aplicación escala de intensidad de fatiga de Krupp, investigación de alteraciones cognitivas mediante batería neuropsicológica repetible breve de Rao (BNR-R) y manifestaciones depresivo-ansiosas mediante cuestionarios de Hamilton, Beck, o ambos. El análisis y versiones ocupadas en BNRR y escalas de Hamilton o Beck están descritos en un trabajo previo nuestro ${ }^{8}$. Revisamos exámenes radiológicos registrando informes, observaciones complementarias y correlato con criterios de Barkhof-Tintoré 9 . Controlamos respuesta a tratamiento, definiendo la falla de este como presencia combinada de alguna de las siguientes condiciones en pacientes con un año de tratamiento:

1) Presencia de brotes.

2) Deterioro de uno o medio punto del puntaje EDSS basal correspondiente.

3) RM con nuevas lesiones en $\mathrm{T} 2$ o lesiones captantes de gadolinio.

Los criterios de envío consistían en:

- Forma clínica EM-RR.
- Diagnóstico según criterios de Mac Donald (2005).

- Presencia de al menos un brote en últimos dos años.

- Menores de 50 años sin uso previo de FIM.

- Enfermedad inferior a 4 años.

- EDSS de 5 ó menos.

Desde diciembre de 2008 se omitieron restricciones de uso de tratamiento previo, edad, tiempo de enfermedad y se amplió la tolerancia de discapacidad hasta EDSS 6.

Al momento de este informe, 218 pacientes tenían seguimiento un año y 132, dos años.

\section{Resultados}

Los pacientes procedían de Chile continental (17o $30^{\prime}$ y $56^{\circ} 30^{\prime}$ latitud sur, $70^{\circ}$ longitud oeste). Hubo 93 que no calificaron para tratamiento las causas se describen en la Figura 1.

De quienes calificaron $67 \%$ eran mujeres (razón 2:1), con inicio promedio de enfermedad a los 29 años ( 5 a 59). El rango etario fluctuó entre 12 y 63 , mediana 35,2 años; $81 \%$ tenía entre 20 y 50 años. El 56\% poseía 13 o más años de escolaridad y $91 \%$ al menos 9 años.

Hubo mayor número de pacientes provenientes de la Región Metropolitana (44\%), coincidente con el mayor porcentaje poblacional de esta. La tasa de envío de pacientes, en relación con la población estimada por región, fluctuó entre 0,53 a 3,25 por 100.000 habitantes, excepción a esto fue Tarapacá desde donde no recibimos pacientes. La tasa más alta de envío fue Atacama con 3,25, seguida por Magallanes con 3,17. No observamos gradiente latitudinal regular y consistente, ni mayor prevalencia de envío de pacientes en relación con el paralelo $40^{\circ}$. Observamos antecedente de otro familiar con EM en 9 pacientes. Si bien no contamos con estudios genéticos, anecdóticamente, de acuerdo a los apellidos del paciente, calificamos 311 casos como mestizos, uno como caucásico y dos como mapuches.

La sintomatología inicial consistió en: alteraciones sensitivas $(20,4 \%)$, motoras $(18 \%)$ y neuritis óptica (NO) (16\%). 27 casos $(8,6 \%)$ debutaron con manifestaciones múltiples. La tasa anual promedio de brotes en los dos años previos fue 1,7. El $56,6 \%$ presentaba hasta 2 brotes por año, 36, $4 \%$ entre 2 y 4 y $7 \%$ más de 4 . El $73 \%$ presentaba 


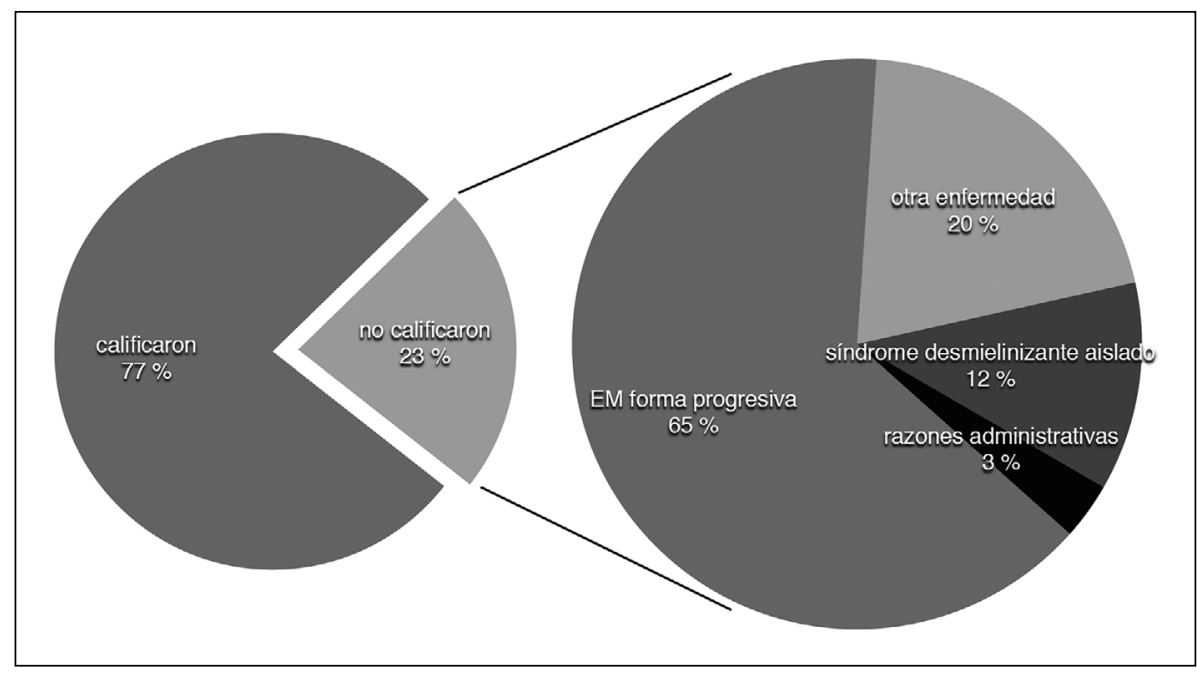

Figura 1. EM-RR en sector público de salud de Chile. Distribución de casos evaluados.
EDSS menor a 4 (35\% de ellos era menor a 2$)$. El $16 \%$ entre 4,5 a 5,5 y $11 \%$ EDSS de 6 o más.

Los porcentajes de depresión, ansiedad y fatiga severa fueron de $41 \%, 31,7 \%$ y $24 \%$ respectivamente.

Consideramos alteración cognitiva (AC) como falla en un sub-test de la BNR-R observándola en 106 pacientes (34\%). Encontramos deterioro cognitivo (DC) en 93 casos (29\%), definido como alteración de dos sub-test de la BNR-R para dominios cognitivos diferentes. Tanto para AC como DC excluimos los casos con fatiga o depresión severa. Los sub-test más alterados fueron los que exploraban atención-concentración y memoria verbal.

En la evaluación de RM, cuantificamos individualmente criterios de Barkhof-Tintoré ${ }^{9,10}$. $\mathrm{Al}$ respecto, $92,9 \%$ presentaba 3 o más de dichas alteraciones.

El estudio de bandas oligoclonales en líquido cefalorraquídeo se realizó en $56,6 \%$ del total, siendo positivas en $44 \%$ de ellos.

Los datos de falla de tratamiento los obtuvimos (según definición explicitada) en evaluaciones de control anuales estandarizadas respecto del ingreso o antes si el médico tratante lo solicitaba. Así, agrupamos los pacientes según tiempo de tratamiento (Tabla 1); en el primer año de control observamos falla en 49 de 218 casos controlados $(22,5 \%)$. En el segundo año la cifra fue de 31 para 121 pacientes $(25,6 \%)$. Hubo 10 casos que presentaron falla a dos FIM, indicados uno de ellos en el primero y otro en el segundo año. Este subgrupo representa 3,2\% del total y $8,3 \%$ de los controlados el segundo año.

Tanto en primero como en segundo año de seguimiento, las condiciones más frecuentes que llevaron a considerar falla de tratamiento fueron combinación de brotes y deterioro de RM con 27 y $38 \%$ de casos para cada año respectivamente. Durante el primer año de seguimiento, 19\% de los pacientes en falla de tratamiento presentaron los tres criterios, el segundo año esta situación se repitió en $29 \%$.

Evaluamos variables clínicas eventualmente predictoras de falla de tratamiento a dos años: tasa de brotes, edad y forma de inicio, nivel EDSS y género. De ellas, las que se relacionaron con mayor probabilidad para dicha condición fueron: género masculino, edad de inicio menor a 40 años y debut con manifestaciones de tronco cerebral (p: 0,05).

Tabla 1. EM-RR en sistema público de salud de Chile. Falla de tratamiento de pacientes al final del primer y segundo año de seguimiento (Tratamiento con Interferón beta 1a, beta $1 \mathrm{~b}$ y Acetato de glatiramer)

\begin{tabular}{|lcc|}
\hline $\begin{array}{l}\text { Pacientes } \\
\text { controlados }\end{array}$ & $\begin{array}{c}\text { Primer año de } \\
\text { tratamiento }\end{array}$ & $\begin{array}{c}\text { Segundo año } \\
\text { de tratamiento }\end{array}$ \\
\hline Total & 218 & 121 \\
Sin falla & $169(77,5 \%)$ & $90(74,4 \%)$ \\
\hline En situación de falla & $49(22,5 \%)$ & $31(25,6 \%)$ \\
\hline
\end{tabular}




\section{Discusión}

Existen escasas publicaciones nacionales respecto de datos clínico-epidemiológicos de pacientes con EM en cualquier modalidad. En 1989, Álvarez y Cárdenas describen conversión a EM en un caso sobre 23 pacientes con $\mathrm{NO}^{11}$. También en 1989, Castillo y cols. evalúan el aporte de la electrofisiología en el diagnóstico de EM en 40 casos con criterios de sospecha diagnóstica; en ese contexto encuentran alta frecuencia de alteración de potenciales evocados somato-sensoriales, algo menor en visuales y escasa en auditivos. Describen como sitios más frecuentemente implicados: nervio óptico, tronco cerebral y médula espinal. Finalmente, plantean que pudiese existir un patrón desmielinizante especial para Chile ${ }^{12}$.

En 1992 Álvarez y cols. describen datos clínicos de 68 pacientes en quienes observan promedio de inicio de la enfermedad 30 años, relación mujeres/ hombres 2:1, manifestaciones motoras como más frecuentes en debut y ausencia de casos familiares. Concluyen que la gravedad de la enfermedad en Chile es similar a otras regiones sin patrón característico ${ }^{13}$.

Nuestro grupo de trabajo describió en al año 2010 características de 79 pacientes con EM-RR evaluados en el programa piloto FONASA-MINSAL de FIM encontrando edad de inicio promedio de 33 años, predominio femenino $(2,4 / 1)$, sintomatología sensitiva como forma inicial aislada más frecuente, sintomatología múltiple como forma más frecuente de presentación y EDSS promedio 3 al momento de la evaluación ${ }^{14}$.
Respecto de la prevalencia en Chile, Fernández y cols., en Magallanes, utilizando método captura-recaptura, la estiman en 14 por 100.000 habitantes ${ }^{15}$. En relación con la incidencia, Díaz y cols., en estudio descriptivo basado en registros de hospitalizaciones por EM, la estiman en $0,9(0,75$ 1,05) casos por 100.000 habitantes en el período 2001-2006. La zona sur presentó tasas de incidencia más altas, siendo catalogada como cluster por los autores y no se encontró correlación entre tasas de incidencia y latitud, o radiación ultravioleta ${ }^{16}$.

En Latinoamérica, una reciente publicación ${ }^{17}$ revisó 38 estudios de diversos países, sumando 4.524 pacientes con diferentes modalidades evolutivas: la forma EM-RR fue más frecuente, el porcentaje de mujeres varió entre 60 y $80 \%$, el principal síntoma inicial fue motor, seguido por NO y sensitivo; la incidencia estimada fluctuó entre 1,6 y 19,6 casos por 100.000 habitantes sin evidenciar gradiente latitudinal. Estos datos, en general, concuerdan con los nuestros con dos salvedades: primero, nuestros pacientes son sólo EM-RR, condición necesaria para ingresar al programa de tratamiento; segundo, al comparar la sintomatología de debut de nuestros pacientes con países de latitud similar como Argentina y Brasil, tenemos algunas diferencias puesto que en ellos es más frecuente el inicio con $\mathrm{NO}$, en tanto que nuestros pacientes presentaron manifestaciones sensitivas como manifestación inicial ${ }^{18-24}$. La Tabla 2 compara algunas características clínicas de nuestros pacientes en relación con otras series de latinoamericanas y latinos de Estados Unidos de Norteamérica.

Tabla 2. EM-RR en sistema público de salud de Chile. Comparación con otras series latinoamericanas

\begin{tabular}{|c|c|c|c|c|c|c|c|c|c|}
\hline $\begin{array}{l}\text { Autor/ } \\
\text { país }\end{array}$ & $\begin{array}{l}\text { Melcon MO/ } \\
\text { Argentina }^{18}\end{array}$ & $\begin{array}{c}\text { Amezcua L/ } \\
\text { USA }^{19}\end{array}$ & $\begin{array}{l}\text { Ferreira/ } \\
\text { Brasil }^{20}\end{array}$ & $\begin{array}{c}\text { Fragoso/ } \\
\text { Brasi }^{21}\end{array}$ & $\begin{array}{c}\text { Moreira MA/ } \\
\text { Brasi }^{22}\end{array}$ & $\begin{array}{l}\text { Arruda/ } \\
\text { Brasi }^{23}\end{array}$ & $\begin{array}{c}\text { Cardoso E/ } \\
\text { Brasi }^{24}\end{array}$ & $\begin{array}{l}\text { Vizcarra/ } \\
\text { Perú }\end{array}$ & $\begin{array}{c}\text { Pacientes } \\
\text { chilenos }\end{array}$ \\
\hline $\begin{array}{l}\text { Número de } \\
\text { pacientes }\end{array}$ & 72 & 125 & 118 & 81 & 302 & 200 & 121 & 55 & 314 \\
\hline $\begin{array}{l}\text { Porcentaje } \\
\text { de EM-RR }\end{array}$ & 75 & 95 & 70 & 82,7 & 72 & 91 & 91,3 & 49 & 100 \\
\hline $\begin{array}{l}\text { Síntoma } \\
\text { inicial más } \\
\text { frecuente }\end{array}$ & $\begin{array}{l}\text { Neuritis } \\
\text { óptica }\end{array}$ & $\begin{array}{l}\text { Neuritis } \\
\text { óptica }\end{array}$ & $\begin{array}{c}\text { No } \\
\text { consignado }\end{array}$ & $\begin{array}{c}\text { No } \\
\text { consignado }\end{array}$ & Sensitivos & $\begin{array}{l}\text { Tronco y } \\
\text { cerebelo }\end{array}$ & $\begin{array}{l}\text { Neuritis } \\
\text { óptica }\end{array}$ & $\begin{array}{l}\text { Neuritis } \\
\text { óptica }\end{array}$ & Sensitivos \\
\hline $\begin{array}{l}\text { Porcentaje } \\
\text { de mujeres }\end{array}$ & 65,3 & 70,3 & 80 & 76,5 & 75 & 64,5 & 80 & 65 & 67 \\
\hline $\begin{array}{l}\text { Edad de } \\
\text { inicio de la } \\
\text { enfermedad }\end{array}$ & 29,3 & 28,4 & 39 & 32 & 29,6 & 32 & 31,1 & 31,5 & 29 \\
\hline
\end{tabular}


Un aspecto menos evaluado en Latinoaméri$\mathrm{ca}^{25,26}$ es la investigación de la función cognitiva y alteraciones psiquiátricas, a este respecto nuestros resultados muestran porcentajes de alteraciones algo inferiores a los principales estudios referentes en este sentido, por ejemplo el multicéntrico RECONEM, realizado en 111 pacientes argentinos evidenció una frecuencia de $43,2 \%$ de alteraciones cognitivas. Creemos que la diferencia se produce porque la definición de DC usada por nosotros es más restrictiva, al considerar el compromiso de dominios cognitivos diferentes, excluir pacientes severamente deprimidos o fatigados y evaluar sólo pacientes de forma EM-RR. Respecto del perfil de compromiso de las mismas, lo encontrado en nuestros pacientes es similar a lo observado tanto en pacientes latinoamericanos como de otras regiones ${ }^{25-27}$.

Lo anterior es más evidente al comparar nuestros resultados con resultados de trabajos como el de Deloire y cols. y el "Cognitive Impairment in Multiple Sclerosis study group" realizados en Europa en los que se evaluaron sólo pacientes EM-RR, con el mismo instrumento y con una definición muy parecida a la nuestra ${ }^{27,28}$. Sobre la presencia de manifestaciones psiquiátricas (depresión o ansiedad), también nuestros resultados son comparables a lo comunicado internacionalmente que sitúa la depresión mayor en torno a $20 \%$ y la ansiedad severa en $50 \%{ }^{29-31}$.

Respecto al acceso a FIM, en la revisión latinoamericana señalada ${ }^{17}$ el uso de ellos fue en promedio $35 \%$, esto representa una diferencia notable con nuestros pacientes ya que todos, una vez evaluados, iniciaron su uso.

En el seguimiento, ante falla de tratamiento, intolerancia o reacciones adversas graves al FIM y ocasionalmente ante respuesta sub-óptima (sólo un criterio de falla), cambiamos la medicación por otro fármaco. Este era también de los denominados de primera línea, ya que nuestro arsenal no contempla hasta ahora la posibilidad de uso de medicamentos de segunda línea de tratamiento ${ }^{32-35}$.

Pese al todavía breve tiempo de seguimiento, los datos de nuestros pacientes nos hacen coincidir con el grupo de Barcelona (Río y cols.) respecto de lo adecuado del uso de criterios combinados (clínicos e imagenológicos) que incluyan la progresión del EDSS en la definición de falla de tratamiento, ya que serían un mejor predictor de discapacidad a largo plazo $^{36}$. Es importante mencionar que aunque existe relativo acuerdo general respecto de los 3 parámetros que seguimos, aún no existe consenso respecto a qué considerar como falla de tratamiento. Algunos centros aplican tolerancia cero a la alteración de cualquier parámetro, incluyendo imágenes en forma aislada, otros consideran que debe estar presente al menos un componente clínico, sea brotes o aumento de discapacidad. Existe discusión sobre el valor del deterioro cognitivo como índice de falla de tratamiento. Los porcentajes de falla de tratamiento de nuestros casos son comparables a lo comunicado internacionalmente sobre estudios basados en práctica clínica ${ }^{37}$. Consideramos que el número de pacientes de nuestra serie resta valor a los datos de falla agrupados según fármacos, información que por lo demás está disponible con respaldo de miles de casos.

Respecto de variables relacionadas a falla de tratamiento a dos años de seguimiento, el género masculino y debut de la enfermedad con lesiones de tronco detectados en nuestro estudio eran predecibles y acorde con la literatura ${ }^{38}$. Sobre el inicio de la enfermedad antes de los 40 años como predictor de falla; si bien no coincide con la literatura, pudiese explicarse porque nuestro criterio de falla considera al menos un elemento de actividad inflamatoria (brotes o nuevas lesiones en RM) y aumento de discapacidad, ello lo hace sensible para la fase inflamatoria de la enfermedad característica de pacientes más jóvenes ${ }^{39}$ y menos útil como instrumento de medición de falla en fases más avanzadas.

Un aspecto destacable de nuestra serie, graficado en la Figura 2, es su carácter nacional. Esto representa un avance en la equidad al acceso a diagnóstico y tratamiento, máxime considerando que el costo directo mensual promedio de los FIM en Chile es aproximadamente US \$ 1.342, siendo el ingreso promedio familiar de nuestros pacientes de US \$ 622. Estos valores se reducen al menos $80 \%$ al ingresar el paciente a tratamiento mediante modalidad de atención institucional en que se enmarca nuestro trabajo.

En suma, el presente trabajo es la continuación natural de la experiencia publicada por nuestro grupo el año $2010^{14}$, constituye hasta ahora la mayor serie nacional al respecto, con una evaluación multidisciplinaria sistemática y periódica. Podemos decir que nuestros pacientes represen- 


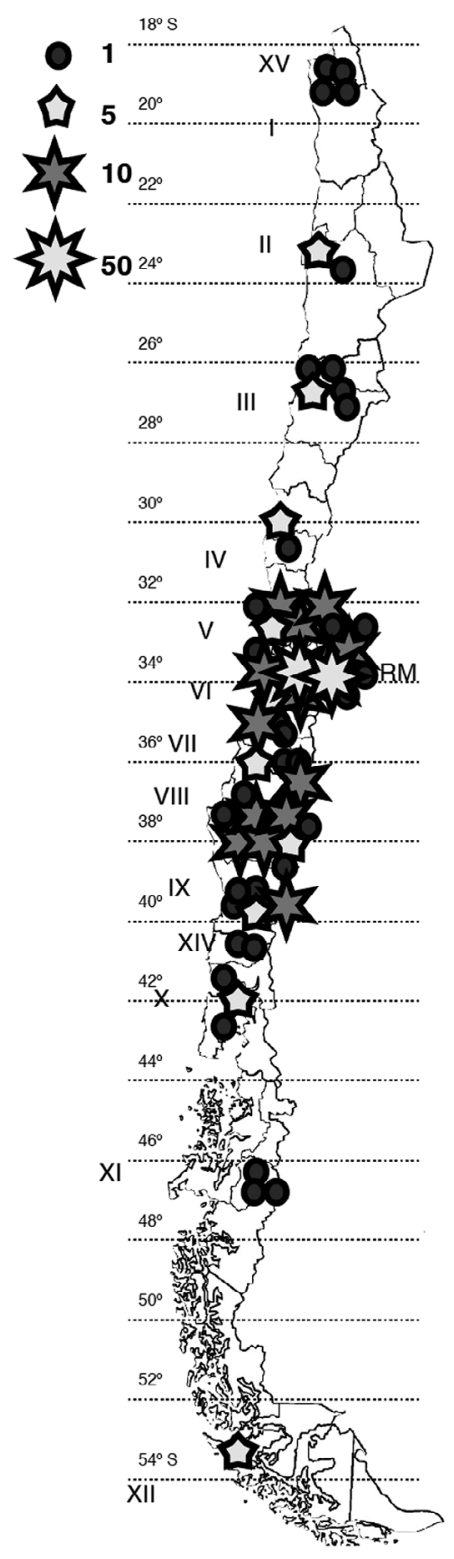

\begin{tabular}{|c|c|c|c|c|}
\hline \multicolumn{5}{|c|}{ CASOS POR REGIÓN Y TASA } \\
\hline \multicolumn{2}{|r|}{ Región } & \multirow{2}{*}{ 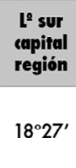 } & \multirow{2}{*}{$\begin{array}{c}\text { NeCasos } \\
4 \\
4\end{array}$} & \multirow{2}{*}{$\begin{array}{c}\text { Tusa }_{100 \mathrm{mi}} \\
\\
2,21\end{array}$} \\
\hline xv & $\begin{array}{c}\text { Arica- } \\
\text { Parinacota }\end{array}$ & & & \\
\hline 1 & Tarapacá & $20^{\circ} 13^{\prime}$ & 0 & 0,00 \\
\hline ॥ & Antofagasta & $23^{\circ} 37^{\prime}$ & 6 & 1,02 \\
\hline IIII & Atacama & $27^{\circ} 22^{\prime}$ & 9 & 3,16 \\
\hline Iv & Coquimbo & $29^{\circ} 58^{\prime}$ & 6 & 0,81 \\
\hline $\mathbf{v}$ & Valparaíso & $33^{\circ} 03^{\prime}$ & 37 & 2,06 \\
\hline RM & Metropolitana & $33^{\circ} 28^{\prime}$ & 139 & 1,98 \\
\hline vı & $O^{\prime}$ Higgins & $34^{\circ} 09^{\prime}$ & 12 & 1,33 \\
\hline vII & Maule & $35^{\circ} 25^{\prime}$ & 17 & 1,66 \\
\hline VIII & Bio-Bio & $36^{\circ} 48^{\prime}$ & 49 & 2,38 \\
\hline $\mathbf{I X}$ & Araucanía & $38^{\circ} 43^{\prime}$ & 18 & 1,82 \\
\hline XIV & De los Rios & $39^{\circ} 49^{\prime}$ & 2 & 0,52 \\
\hline$x$ & De los Lagos & $41^{\circ} 28^{\prime}$ & 7 & 0,82 \\
\hline $\mathbf{x} \mathbf{I}$ & Aysén & $45^{\circ} 34^{\prime}$ & 3 & 2,81 \\
\hline xII & Magallanes & $53^{\circ} 09^{\prime}$ & 5 & 3,13 \\
\hline \multicolumn{3}{|c|}{ Total } & 314 & 1,80 \\
\hline
\end{tabular}

Figura 2. EM-RR en el sector publico de Chile. Distribución territorial de envío de casos.

tan un grupo de portadores de EM-RR con poca discapacidad física pero con enfermedad muy activa clínicamente aún bajo tratamiento con FIM. Básicamente, como grupo no se diferencian en términos demográficos y clínicos (físico, cognitivo y psiquiátrico) de lo descrito en nuestro informe anterior, por otros autores latinoamericanos o de otras regiones. El debut de la enfermedad por manifestaciones sensitivas sería una diferencia a señalar de nuestros casos respecto de la mayoría de series latinoamericanas.

En relación a la efectividad de los FIM de primera línea como los que disponemos, lo que hemos observado en nuestros pacientes también concuerda con lo comunicado internacionalmente $^{37}$. 
Respecto de la evaluación multidisciplinaria en $\mathrm{EM}$, diversas publicaciones ${ }^{40-42}$ señalan que esta forma de trabajo redunda en una atención más adecuada, con mejor impacto en la calidad de vida de los pacientes, que la exclusiva atención médica. La multiplicidad de problemas de los pacientes requiere terapias complejas integradas e integrales, haciendo necesario la interacción de un equipo para su manejo, la alternativa de evaluación secuencial independiente nos parece menos efectiva, por ello consideramos de máxima importancia la mantención de un equipo amplio de trabajo e idealmente su replicación en otras zonas de Chile.

\section{Referencias}

1. Kurtzke JF. Epidemiology and multiple sclerosis. Phys Med Rehabil Clin N Am 2005; 16 (2): 327-49.

2. Koch-Henriksen N, Sørensen PS. The changing demographic pattern of multiple sclerosis epidemiology. Lancet Neurol 2010; 9 (5): 520-32.

3. Hogancamp W, Rodríguez M, Weinshenker B. The epidemiology of multiple sclerosis. Mayo Clin Proc 1997; 72: $871-8$.

4. Gasperini C. Differential diagnosis in multiple sclerosis. Neurol Sci. 2001; 22 Suppl 2: S93-7.

5. Confavreux C, Vukusic S, Adeleine P. Early clinical predictors and progression of irreversible disability in multiple sclerosis: an amnesic process. Brain 2003; 126 (Pt 4): 770-82.

6. Abad P, Nogales-Gaete J, Rivera V, Cristiano E, Hamuy F, Oehninger C, et al. Grupo de Estudio de Esclerosis Múltiple de Latinoamérica Lactrims. Documento de consenso de LACTRIMS para el tratamiento farmacológico de la esclerosis múltiple y sus variantes clínicas. Rev Neurol 2012; 55 (12): 737-48.

7. Vizcarra-Escobar D, Cava-Prado L, Tipismana-Barbarán M. Esclerosis múltiple en Perú. Descripción clínico epidemiológica de una serie de pacientes. Rev Neurol 2005; 41 (10): 591-5.

8. Nogales-Gaete J, Aracena R, Díaz V, Zitko P, Eloiza C, Cepeda-Zumaeta $S$, et al. Evaluación neuropsicológica en 129 pacientes chilenos con esclerosis múltiple recurrente remitente previo a inicio de fármacos inmunomoduladores. Rev Med Chile 2012; 140 (11): 1437-44.

9. Barkhof F, Filippi M, Miller DH, Scheltens P, Campi A, Polman $\mathrm{CH}$, et al. Comparison of MR imaging criteria at first presentation to predict conversion to clinically definite multiple sclerosis. Brain 1997; 120: 2059-69.

10. Tintoré M, Rovira A, Martínez MJ, Río J, Díaz-Villosla- da $\mathrm{P}$, Brieva $\mathrm{L}$, et al. Isolated demyelinating syndromes: comparison of different MR imaging criteria to predict conversion to clinically definite multiple sclerosis. AJNR Am J Neuroradiol 2000; 21 (4): 702-6.

11. Álvarez G, Cárdenas M. Multiple sclerosis following optic neuritis in Chile. J Neurol Neurosurg Psychiatry 1989; 52 (1): 115-7.

12. Castillo JL, Ruíz F, Lavados J, Álvarez G, Monari M. La esclerosis múltiple en nuestro medio asistencial: análisis clínico, electrofisiológico e inmunológico. Rev Med Chile 1989; 117 (10): 1122-9.

13. Álvarez G, Castillo JL, Ruiz F, Cárdenas M, Fauré E, Lavados J. Multiple sclerosis in Chile. Acta Neurol Scand 1992; 85 (1): 1-4.

14. Nogales-Gaete J, Aracena R, Agurto P, Cepeda S, Figueroa C, González Carola, et al. Programa piloto para pacientes beneficiarios de Fonasa, que padecen Esclerosis Múltiple. Tratamiento con Inmunomoduladores en el Sistema Público de Salud de Chile. Informe del primer año, 10 de julio 2008-30 de junio de 2009. Rev Chil Neuro-Psiquiat 2010; 48 (supl. 1): 9-92.

15. Fernández R. Esclerosis Múltiple en la Región de Magallanes-Chile, Extremo Austral del Continente en: Arriagada C, Nogales-Gaete J. Edit. Esclerosis Múltiple: Una mirada Ibero-panamericana, Segunda Edición, New York, Demos Medical Pub 2008, PP 229-36.

16. Díaz V, Barahona J, Antinao J, Quezada R, Delgado I, Silva C, et al. Incidence of multiple sclerosis in Chile. A hospital registry study. Acta Neurol Scand 2012; 125: 71-5.

17. Ojeda E, Díaz-Cortés D, Rosales D, Duarte-Rey C, Anaya JM, Rojas-Villarraga A. Prevalence and clinical features of multiple sclerosis in Latin America. Clin Neurol Neurosurg 2013; 115 (4): 381-7.

18. Melcon MO, Gold L, Carrá A, Cáceres F, Correale J, Cristiano E, et al. Patagonia Multiple Sclerosis Research Project. Argentine Patagonia: prevalence and clinical features of multiple sclerosis. Mult Scler 2008; 14 (5): 656-62.

19. Amezcua L, Lund BT, Weiner LP, Islam T. Multiple sclerosis in Hispanics: a study of clinical disease expression. Mult Scler J 2011; 17: 1010-6.

20. Ferreira M, Machado M, Vilela M, Guedes M, J. Ataíde L, Santos S, et al. Epidemiology of 118 cases of multiple sclerosis after 15 years of follow-up on the reference center of Hospital da Restauracão, Recife, Pernambuco, Brazil. Arquivos de Neuro-Psiquiatria 2004; 62: $1027-$ 32.

21. Fragoso YD, Fiore APP. Description and characteristics of 81 patients attending the Reference Center for Multiple Sclerosis of the coastal region of the state of São 
Paulo-Brazil. Arquivos de Neuro-Psiquiatria 2005; 63: 741-4.

22. Moreira MA, Felipe E, Mendes MF, Tilbery CP. Multiple sclerosis: descriptive study of its clinical forms in 302 cases. Arquivos de Neuro-Psiquiatria 2000; 58: 460-6.

23. Arruda WO, Scola RH, Teive HA, Werneck LC. Multiple sclerosis: report on 200 cases from Curitiba, Southern Brazil and comparison with other Brazilian series. Arquivos de Neuro-Psiquiatria 2001; 59: 165-70.

24. Cardoso E, Fukuda T, Pereira J, Seixas J, Miranda R, Rodrigues B, et al. Clinical and epidemiological profile of multiple sclerosis in a reference center in the State of Bahia, Brazil. Arq Neuropsiquiatr 2006; 64 (3B): 727-30.

25. Cáceres F, Vanotti S, Rao S; RECONEM Workgroup. Epidemiological characteristics of cognitive impairment of multiple sclerosis patients in a Latin American country. J Clin Exp Neuropsychol 2011; 33 (10): 1094-8. Epub 2011 Oct 6. 15.

26. Sepulcre J, Vanotti S, Hernández R, Sandoval G, Cáceres F, Garcea $\mathrm{O}$, et al. Cognitive impairment in patients with multiple sclerosis using the Brief Repeatable Battery-Neuropsychology test. Mult Scler 2006; 12 (2): 187-95.

27. Deloire M, Ruet A, Hamel D, Bonnet M, Brochet B. Early cognitive impairment in multiple sclerosis predicts disability outcome several years later. Mult Scler 2010; 16 (5): 581-7.

28. Patti F, Amato MP, Trojano M, Bastianello S, Tola MR, Goretti B, et al. COGIMUS Study Group. Cognitive impairment and its relation with disease measure in mildly disabled patients with relapsing-remitting multiple sclerosis: baseline results from the Cognitive Impairment in Multiple Sclerosis (COGIMUS) study. Mult Scler 2009; 15 (7): 779-88.

29. Feinstein A. Multiple sclerosis and depression. Mult Scler 2011; 17 (11): 1276-81.

30. Wood B, van der Mei IA, Ponsonby AL, Pittas F, Quinn S, Dwyer T, et al. Prevalence and concurrence of anxiety, depression and fatigue over time in multiple sclerosis. Mult Scler 2013; 19 (2): 217-24.

31. Giordano A, Granella F, Lugaresi A, Martinelli V, Trojano $\mathrm{M}$, Confalonieri $\mathrm{P}$, et al. SIMS-Trial group. Anxiety and depression in multiple sclerosis patients around diagnosis. J Neurol Sci 2011; 307 (1-2): 86-91.

32. Freedman MS, Blumhardt LD, Brochet B, Comi G, Noseworthy JH, Sandberg-Wollheim M, et al. Paris Workshop Group. International consensus statement on the use of disease-modifying agents in multiple sclerosis. Mul Scler 2002; 8 (1): 19-23.

33. García-Merino A, Fernández O, Montalbán X, de Andrés $\mathrm{C}$, Oreja-Guevara $\mathrm{C}$, Rodríguez-Antigüedad A, et al. Documento del Grupo de Consenso de la Sociedad Española de Neurología sobre el uso de medicamentos en esclerosis múltiple. Neurología 2013; 28 (6): 375-8.

34. García Merino A, Fernández O, Montalbán X, de Andrés C, Arbizu T. Documento de consenso de la Sociedad Española de Neurología sobre el uso de medicamentos en esclerosis múltiple: escalado terapéutico. Neurología 2010; 25 (6): 378-90.

35. Chile. Ministerio de Salud. Guía Clínica Esclerosis Múltiple. 2010.

36. Río J, Nos C, Tintoré M, Téllez N, Galán I, Pelayo R, et al. Defining the response to interferon-beta in relapsingremitting multiple sclerosis patients. Ann Neurol 2006; 59 (2): 344-52.

37. Pozzilli C, Prosperini L, Sbardella E, De Giglio L, Onesti E, Tomassini V. Postmarketing survey on clinical response to interferon beta in relapsing multiple sclerosis: the Roman experience. Neurol Sci 2005; 26 Suppl 4: S174-8.

38. Confavreux C, Vukusic S. The clinical epidemiology of multiple sclerosis. Neuroimaging Clin N Am 2008; 18 (4): 589-622.

39. Vukusic S, Confavreux C. Natural history of multiple sclerosis: risk factors and prognostic indicators. Curr Opin Neurol 2007; 20 (3): 269-74.

40. Cheng EM, Crandall CJ, Bever CT Jr, Giesser B, Haselkorn JK, Hays RD, et al. Quality indicators for multiple sclerosis. Mult Scler 2010; 16 (8): 970-80.

41. Wade DT. Cognitive assessment and neurological rehabilitation. Clin Rehabil. 2002; 16 (2): 117-8.

42. Guo ZN, He SY, Zhang HL, Wu J, Yang Y. Multiple sclerosis and sexual dysfunction. Asian J Androl 2012; 14 (4): 530-5. 